\title{
Study of the Types of Domestic Violence Committed Against Women Referred to the Legal Medical Organization in Urmia - Iran
}

\author{
Nader Aghakhani, ${ }^{1}$ Hamid Sharif Nia, ${ }^{2}$ Ehsan Moosavi, ${ }^{3}$ Ali Eftekhari, ${ }^{3}$ Abbas Zarei, ${ }^{3}$ Nasim \\ Bahrami, ${ }^{4}$ and Ali Reza Nikoonejad ${ }^{5, "}$ \\ ${ }^{1}$ Department of Medical Surgical Nursing, School of Nursing and Midwifery, Urmia University of Medical Sciences, Urmia, IR Iran \\ ${ }_{2}^{2}$ School of Nursing and Midwifery of Amol, Mazandaran University of Medical Sciences, Sari, IR Iran \\ ${ }^{3}$ Legal Medicine Organization, Urmia, IR Iran \\ 4 Social Determinants of Health Research Center, Qazvin University of Medical Sciences, Qazvin, IR Iran \\ 5 Department of Infectious Diseases, School of Medicine, Urmia University of Medical Sciences, Urmia, IR Iran \\ ${ }^{*}$ Corresponding author: Ali Reza Nikoonejad, Department of Infectious Diseases, School of Medicine, Urmia University of Medical Sciences, Urmia, IR Iran. Tel: +98-4412754916, \\ Fax:+98-4412754916, E-mail: nikoonejad.anikoo@gmail.com
}

Received 2014 August 16; Revised 2014 December 24; Accepted 2015 May 22.

\begin{abstract}
Background: Today, domestic violence against women is a growing epidemic that can be observed in many countries.

Objectives: This study was carried out to determine the types of domestic violence against women who were referred to the Legal Medical Organization of Iran in Urmia, Iran in 2012.

Materials and Methods: The descriptive survey included demographic information, abuse screening, and items regarding partner involvement. Data was gathered using face-to-face structured interviews. The study population included 300, women 18 years of age or older, and data was collected about their demographic characteristics and the types of domestic violence they experienced. SPSS software version 16 was used for the analyses.

Results: The majority of participants were in the $25-30$ age group, and $83 \%$ of them were battered by their husbands in various ways. No significant relationships were observed between violence and unemployment, increasing age, and home ownership.

Conclusions: The prevalence of abuse reported by women in this population suggests that many women that are referred to the Legal Medical Organization of Iran may have a history of abuse. Abused women may have different reasons for seeking a divorce. If routine screening for abuse is included in counseling, health providers will have the opportunity to develop a safety plan and initiate appropriate referrals.
\end{abstract}

Keywords: Abused Women, Domestic Violence, Legal Medical Organization of Iran

\section{Background}

The comprehensive study on domestic violence (DV) conducted by the World Health Organization (WHO) indicated that DV has probably always been part of the individual experience of women, and it results in physical, sexual, or mental injury or suffering (1). Domestic violence is observed in many countries and it can be a public health problem (2). It consists of physical, sexual (forcing undesired sexual behavior by one person upon another), economic (having control over the other partner's access to economic resources), and psychological (actions aimed to dominate and manipulate the partner) aspects caused by many factors $(3,4)$. Violence against a woman results in her isolation. Victims can be of any ethnicity, age, level of education, or income level. Some of the factors that have been associated with domestic violence in Iran include women's education, prior experience of DV, illicit sexual relations, and age difference. There is a significant positive relationship between women's eco- nomic situation and psychological, physical, and social violence. In order to promote preventive strategies for the consequences of $\mathrm{DV}$, it is essential to identify the potential risk factors for it $(5,6)$.

Women are subjected to domestic violence significantly more often than men; this is true for all societies, and it is a universal, trans-historical, and trans-cultural problem as old as human history (7). Domestic violence is one of the causes of disability and death in women, and it is related to the status of women who live in a culture that considers males to be the dominant sex and that victimizes females. Women, who have children, suffer from a lower level DV (8).

WHO has defined DV as behavior within an intimate relationship that causes the physical, sexual, or psychological abuse of women (9). Women who are abused often have a range of physical health problems, including being rundown, aching all over, having headaches, increased hyper-

Copyright (C) 2015, Mazandaran University of Medical Sciences. This is an open-access article distributed under the terms of the Creative Commons Attribution-NonCommercial 4.0 International License (http://creativecommons.org/licenses/by-nc/4.0/) which permits copy and redistribute the material just in noncommercial usages, provided the original work is properly cited. 
tension, chronic fatigue, and gastrointestinal problems. These health problems dramatically reduce their ability to cope with the violence they experience (10). These health problems result in chronic physical diseases, and reduced practice of healthy actions and healthy lifestyle choices (11). Violent behavior against women is seen in all societies and at all socioeconomic levels. Economic problems are the most common cause of DV and studies have shown a significant relationship between low socioeconomic levels and a higher risk of violence (12).

While there is a great deal of information about the social implications of DV, its impact must be taken into consideration and society must be informed about this critical issue (13).

\section{Objectives}

This study aimed to evaluate the types of DV against women who were referred to the Legal Medical Organization of Iran, located in Urmia, Iran, in 2012.

\section{Materials and Methods}

A total of 300 women who were referred to the Legal Medical Organization of Iran because of DV, participated in this cross-sectional study. The sample size was calculated based on the prevalence of DV in Iran with an absolute precision of 0.05 and a confidence level (CI) of 95\%. The study was powered at $80 \%$ with a two-sided $5 \%$ to achieve a statistical significance on a moderate standardized effect size of 0.4. Required data were collected through faceto-face interviews using a structured questionnaire. The questionnaire contained 32 questions about all types of DV that the participants experienced, and their personal and family information was collected. The research team initially developed the questionnaire after an extensive review of the relevant literature to achieve good content validity. The validity of the questionnaire was confirmed by content validity. Its reliability with internal consistency was confirmed; the Cronbach's alpha calculation was 0.85 and the test-retest reliability was 0.88 . All of the collected data were considered to be confidential and were handled only by the researchers.

\subsection{Ethical Issue}

The study was approved by the Ethics Committee and written informed consent was obtained from all of the participants.

\section{2. Statistical Analysis}

Basic descriptive statistics were presented using mean $( \pm S D)$ for quantitative variables and $n(\%)$ for qualitative variables. To determine the association between DV and demographic variables, Student's independent t-test and one-way analysis of variance (ANOVA) were used. The database was exported to SPSS for Windows (version 16; SPSS Inc., Chicago, IL, USA) and analysis was performed. The prevalence of different forms of DV for each phrase was computed with $95 \% \mathrm{CI}$. A probability level of $\mathrm{P}<0.05$ was determined as statistically significant.

\section{Results}

In the present study, the mean age of the 300 female participants was 29.20 ( \pm 7.52 ). Inclusion criteria consisted of the place of residence, either in the city of Urmia, Iran or in rural areas, and being married. No exclusion criteria were considered. Among the participants, 277 (92.3\%) lived in the city of Urmia and 23 (7.7\%) lived in rural areas. Moreover, 24 women (8\%) had consanguineous marriages before their marriage and 33 (11\%) had a history of previous marriages. As indicated in Table 1, differences were found in the level of education of the men and women and the women's family.

Furthermore, 47 women (15.7\%) and 167 men (55.7\%) had a steady job. Different forms of violence were commitment against women 4.98 ( \pm 0.33) times per month (based on their self-reported statements). The mean age of marriage was 21.5 ( \pm 6.51) years (max: 40 and minnutes: 12 years) and the mean age gap between a wife and a husband was 6.1 ( \pm 0.31 ) years. In addition, 31 couples (10.3\%) had married to resolve tribal and familial disputes. Of the men, 57 (19\%) had been previously married and 38 (12.7\%) had more than one wife. In 11 cases (3.7\%), the women were older than the men. Furthermore, 230 couples (73.7\%) had children, and 38 women (12.7\%) were forced to marry (based on their selfreported statements). In 218 cases (72.7\%), both the men and the women desired to get married, in 59 cases (19.7\%) the men desired to get married, and in 20 cases (6.7\%) the women desired to get married. Moreover, 99 couples (33\%) owned a home and 150 couples (50\%) rented a home, and other couples lived with their family members. A history of violence during the engagement period was observed in 82 women (27.3\%), and 78 women (26\%) had been violated by someone else in the husband's family. In $49.7 \%$ of the women and $71.3 \%$ of the men their parents' emotional relationship was not satisfactory. This was evaluated based on the question: "How was the emotional relationship between your spouse's parents?"

Methods of coping with violence in women were: crying (24\%), fighting (7\%), seeking refuge at her father's house (5\%), and forgiveness (2\%); however, most of the women (62\%) did not take any action. In addition, 251 women (83.7\%) were referred to the Legal Medical Organization of Iran for the first time, while others had been referred several times. As indicated in Table 2, the causes of DV varied.

In 99 cases (33\%), the couples had known each other before marriage. Moreover, 39 women (13\%) and 42 men (14\%) were substance abusers, and 153 men (51\%) were cigarette smokers. As indicated in Table 3, most of the women had experienced physical, sexual, economic, and psychological violence.

A significant relationship was observed between type of lodging and physical $(\mathrm{P}=0.42)$, economic $(\mathrm{P}=0.57)$, and sex- 
ual violence $(\mathrm{P}=0.58)$. No significant relationship was found between consanguineous marriages and physical violence $(\mathrm{P}=0.38)$. However, the relationship between consanguineous marriage $(P=0.003)$, economic violence $(P=0.052)$, and sexual violence was significant $(P=0.003)$. The rate of physical violence was significantly higher in men without a steady occupation $(\mathrm{P}<0.001)$. Nevertheless, no significant relationship was found between the men's occupation and economic $(\mathrm{P}=0.147)$ and sexual violence $(\mathrm{P}=0.48)$.

No significant relationship was found between marriage with the purpose of resolving tribal and familial disputes and physical $(P=0.93)$, economic $(P=0.96)$, and sexual violence $(P=0.312)$.

Our results showed that the rate of sexual violence was significantly higher in older men $(P=0.042)$. No significant relationship was found between the men's age and economic $(P=0.34)$ and physical violence $(P=0.19)$. No significant relationship was found between running away from home with one's husband and physical ( $\mathrm{P}=$ $0.97)$, economic $(P=0.83)$, and sexual violence $(P=0.13)$.

The rate of physical violence was higher in women without family support, but it was not statistically significant $(P=0.25)$. Furthermore, no significant relationship was found between family support and economic $(P=0.67)$ and sexual violence (0.78).

Our study demonstrated that the rate of physical violence was significantly lower in women who had a blood relationship with their husband before marriage $(\mathrm{P}=$ 0.012 ). Moreover, there was no significant relationship between economic $(P=0.36)$ and sexual violence $(P=0.64)$ and dating before marriage. There was no significant relationship between physical $(\mathrm{P}=0.21)$ and economic violence $(\mathrm{P}=0.178)$ and women's hijab. A significant positive relationship was found between the husband's drug abuse and physical $(\mathrm{P}=0.042)$ and economic violence $(\mathrm{P}$ $=0.036)$. However, no significant relationship was found between drug abuse and sexual violence $(\mathrm{P}=0.35)$.

A significant negative relationship was found between the husband's education and physical violence $(\mathrm{P}=0.001)$. The rate of sexual $(P=0.21)$ and economic violence $(P=0.07)$ was higher in women with low-educated husbands, but there was no significant relationship between these variables.

Physical violence was significantly higher in low-income couples without a home $(\mathrm{P}=0.011)$. There was no significant relationship between economic $(\mathrm{P}=0.11)$ and sexual violence $(\mathrm{P}=0.21)$ and the couples' living situation and income, although both types of violence were higher in those women. The rate of sexual $(\mathrm{P}=0.033)$ and physical violence $(P=0.021)$ was significantly higher in men without faith.

However, no significant relationship was found between economic violence and the husband's faith ( $\mathrm{P}=$ 0.95). In addition, there was no significant relationship between the age at marriage and physical $(P=0.43)$, economic $(P=0.45)$, and sexual violence $(P=0.43)$.

\begin{tabular}{lcccc}
\hline \multicolumn{1}{l}{ Table 1. Level of Education of Men and Women and the Women's Family } \\
\hline Level of Education & Wife & Husband & Women's Father & Women's Mother \\
\hline Illiterate & $9(3)$ & $6(2)$ & $155(51.7)$ & $183(61)$ \\
Elementary school & $49(16.3)$ & $100(33.3)$ & $80(26.7)$ & $63(21)$ \\
Guidance & $80(26.7)$ & $60(20)$ & $30(10)$ & $17(5.7)$ \\
\hline High school & $113(37.7)$ & $98(32.7)$ & $20(6.7)$ & $21(7)$ \\
\hline BSc b & $41(13.7)$ & $25(8.3)$ & $28(9.3)$ & $3(1)$ \\
MSc c & $8(2.7)$ & $9(3)$ & NA & NA \\
\hline PhD ${ }^{\text {d }}$ & NA & $2(0.7)$ & & NA \\
\hline
\end{tabular}

Abbreviations: NA, not available.

${ }^{\mathrm{a}}$ Data are presented as No. (\%).

$\mathrm{b}$ Bachelor of Science.

c Master of Science.

$\mathrm{d}_{\text {Doctor of Philosophy. }}$

\begin{tabular}{lc}
\hline Table 2. Causes of Domestic Violence & No. (\%) \\
\hline Parametr & $3(1)$ \\
\hline Psychological disease history & $137(45.7)$ \\
\hline Hates her husband & $2(0.7)$ \\
\hline Liaison marriage (having a relationship before marriage) & $67(22.3)$ \\
\hline Running away from home with one's husband & $90(30)$ \\
\hline Continued living with husband & $3(1)$ \\
\hline Woman's infertility & $12(3)$ \\
\hline Man's infertility & $9(3)$ \\
\hline Stepfather & $20(6.7)$ \\
\hline Stepmother & \\
\hline
\end{tabular}


Aghakhani N et al.

\begin{tabular}{|c|c|}
\hline Violence Type & Frequency No.(\%) \\
\hline Psychological violence & $298(99.2)$ \\
\hline Vilification & $235(77.9)$ \\
\hline Humiliate & $225(74.5)$ \\
\hline Intense reaction & $184(61.3)$ \\
\hline Treats of divorce & $185(61.3)$ \\
\hline Decisions without consulting & $207(68.7)$ \\
\hline Jape & $195(64.6)$ \\
\hline Cavil & $225(74.4)$ \\
\hline Treats of remarriage & $203(67.3)$ \\
\hline Sexual violence & $221(73.4)$ \\
\hline Sex any time he wanted & $112(37.3)$ \\
\hline Boring sex & $113(37.7)$ \\
\hline Leave wife alone after orgasm & $171(57)$ \\
\hline Sex in unusual way & $115(38.3)$ \\
\hline Disregarding to of sexuality & $152(50.2)$ \\
\hline Economic al violence & $262(87.3)$ \\
\hline Prevent economic independence & $156(52)$ \\
\hline Spending money onfor friends & $136(45.3)$ \\
\hline Being a miserly husband & $90(30)$ \\
\hline Control spending obsessively & $112(37.3)$ \\
\hline Husband get takes their income by force & $34(11.3)$ \\
\hline Physical violence & $268(89.3)$ \\
\hline Slap in the face & $234(78)$ \\
\hline Strangling & $171(57)$ \\
\hline Thrash & $212(70.7)$ \\
\hline Pushing & $212(70.7)$ \\
\hline Kicking & $240(80)$ \\
\hline Pulling hair & $195(65)$ \\
\hline
\end{tabular}

\section{Discussion}

This study identified the areas of health most consistently related to experiences of DV across a wide range of samples, and women with a history of experiencing DV also experienced significantly poorer functional and somatic physical health.

It appears that DV is a common health problem that results in prolonged posttraumatic physical and psychological disorders. The present study also showed that false traditional beliefs, low educational level, lack of knowledge about women's rights, and low social support for abused women impact the continuation of complications from DV. In a study in Iran on 40 battered women that were referred to the local Legal Medical Organization, depression, anxiety, and stress were significantly higher in the abused wives as compared to the control group (14). A higher rate of depression, anxiety, and phobias was observed in women who were abused by their husbands than in non-abused women (15).

In our study, the incidence of sexual, physical, economic, and psychological violence was high. In another study, the incidence of sexual and physiological violence were $22 \%$ and $32 \%$, respectively (16). In the study by Weingourt et al., the incidence of physical, sexual, and physiological violence was $32 \%, 23 \%$, and $60 \%$, respectively (17). In the study by Avdibegovic and Sinanovic, the incidence of sexual, physical, and physiological violence was $43.5 \%, 63.2 \%$, and $69.6 \%$, respectively (18).

In this present study, the prevalence of DV varied, based on the geographic areas studied. Women living in urban areas had the highest prevalence, while women in the city of Urmia had the lowest. This finding could mean that women in urban areas have more knowledge about their rights, and more women living in cities report DV 
events in comparison to women in rural areas. No similar significant difference was found.

According to our findings, higher unemployment, a lower level of education, and lower socioeconomic status aggravated a person's DV status. On the other hand, based on cultural standards and lack of financial independence, women in rural areas face more resistance in obtaining a divorce from their traditional society.

Based on the findings of our study, the rate of domestic physical violence was higher in women with a lower level of education compared to more educated women. This might be due to educated women coping more effectively with violence and having a better economic situation. Another study showed that unemployment of women is higher, whereas rural residence had a greater impact on psychological and sexual violence (19).

Our study demonstrates a significant relationship between physical violence and the husband's employment status, drug abuse, level of education, and dwelling situation. One study demonstrated that physical violence has a significant association with the employment status, residence (rural or urban), age and occupation of women, and monthly family income (20). Rosen et al. showed that the rate of committing violence against women was higher in men with low income, low education, and physical and mental fatigue (21). Bonomi et al. also observed a significant relationship between violence and low income (22). Our study obtained a similar result.

Another research study on women showed that the rate of domestic violence is significantly correlated with factors, such as the husband's level of education, economic condition, employment, and addiction status, as well as geographical setting (23).

According to one other study, age, education, occupation, marital duration, and the husband's addiction appeared to be significant factors associated with all types of DV, and level of family income was a highly protective factor against the occurrence of violence (24).

However, culture may have a more significant impact than geographic region. In some Islamic countries, studies showed that at least one-third of the women experience physical violence by their husbands (2).

In this present study, culture impacted the prevalence of domestic physical violence. Some traditional societies have negative attitudes toward women's roles and rights. One study found a significant association between the husband's level of education and his preference for a male child, and the prevalence of DV (25).

Ghahhari et al. reported that $73.5 \%, 92.2 \%$, and $49.6 \%$ of women in the general population of Sari (north of Iran) were subjected to physical, emotional, and sexual abuse, respectively, at least once during their lives. They also reported that most of the women suffered from mild degrees of spousal abuse and an association was found between spousal abuse and the number of offspring, the husband's age, unemployment, low level of education, drug abuse, and mental disorders (26). Pournaghash
(2011) found that the prevalence of wife beating was lower only when both partners were educated (27), which confirms our study's results that a higher level of education is associated with a decrease in DV.

The present study found a significant relationship between sexual violence and the husband's age and the lack of men's faithfulness. It also showed a significant relationship between sexual violence and setting, age, and the educational level of women. In this study, economic violence was found to have a significant relationship with the husband's education level and drug abuse.

According to this study, acceptance of domestic physical violence against wives was higher among women who had experienced abuse. It is interesting that, in another study, $23 \%$ of the women stated that their husbands had the right to commit different kinds of DV; that study found that some socio-economic characteristics of women have a significant association with DV (27).

For this research, we conducted a large-scale field study of 300 Iranian women in Urmia, Iran. Our results showed that higher education, employment, and a good economic situation could help significantly reduce DV. Convincing husbands to allow their wives to participate in decision making is another important step that could help reduce the prevalence of DV. Increasing public awareness of human rights through education is a very important measure that should be taken by reliable local officials. Future studies also need to focus on the pathways to recovery from experiences of DV and how health services can play a role in rehabilitative actions. It is clear that DV has detrimental consequences for an individual's well-being. The findings of this study affirm that DV is a public health problem and a legal and social issue.

This study had several limitations that could impact the generalizability of its results. First, because this is a correlational study the authors cannot infer cause and effect. Second, it is possible that some of the women might not have been truthful in their answers to the DV questionnaire due to cultural problems in Iran. Finally, women that participated in this study were under a lot of stress, which limits the generalizability of the findings to other populations.

In summary, since the present research has merely focused on the DV problems of women who were referred to the Legal Medical Organization of Iran, additional studies are needed to examine DV in other groups of women.

\section{Acknowledgments}

The authors would like to thank the manager and staff at the Urmia Legal Medical Organization, and all the people who kindly helped by providing us with the opportunity to conduct this study.

\section{Authors' Contributions}

Nader Aghakhani, Hamid Sharif Nia, conceived and designed the study, collected and interpreted the clinical data, and then drafted and revised the manuscript; Ehsan Moosavi, Ali Eftekhari, and Abbas Zarei, participated in 
data gathering and helped interpret the clinical data; they also helped draft and revise the manuscript; Nasim Bahrami, helped conceive and design the study and assisted in drafting and revising the manuscript; Ali Reza Nikoonejad, conceived and designed the study, collected and interpreted the clinical data, conducted the statistical analysis, and critiqued and revised the manuscript to ensure the intellectual strength of its content; All of the authors read and approved the final manuscript.

\section{Declaration of Interest}

None declared.

\section{References}

1. Al-Tawil NG. Association of violence against women with religion and culture in Erbil Iraq: a cross-sectional study. BMC Public Health. 2012;12:800. doi:10.1186/1471-2458-12-800. [PubMed: 22984840]

2. Garcia-Moreno C, Jansen HA, Ellsberg M, Heise L, Watts $\mathrm{CH}$, W.H.O. Multi-country Study on Women's Health. et al. Prevalence of intimate partner violence: findings from the WHO multicountry study on women's health and domestic violence. Lancet. 2006;368(9543):1260-9. doi: 10.1016/S0140-6736(06)69523-8. [PubMed:17027732]

3. Adams AE, Sullivan CM, Bybee D, Greeson MR. Development of the scale of economic abuse.Violence Against Women. 2008;14(5):56388. doi: 10.1177/1077801208315529. [PubMed:18408173]

4. Rosenthal AM, Fridell LA, Dantzker ML, Fisher-Stewart G, Saavedra PJ, Makaryan T, et al. Community Policing: Then and Now. NIJ Journal. 2003;(249):34.

5. Ansara DL, Hindin MJ. Psychosocial consequences of intimate partner violence for women and men in Canada. $J$ Interpers Violence. 2011;26(8):1628-45. doi: 10.1177/0886260510370600. [PubMed: 20501897]

6. Salimi SH, Azad-Marzabadi E, Karaminia R, Mirzamani SM, Hosseini-Sangtrashani SA. Being a wife of a veteran with psychiatric problem or chemical warfare exposure: A preliminary report from Iran. Iranian j psychiatry. 2006;1(2):65-9.

7. Molavardi Sh, Khatiblou F, Ashtari B, Tabatabai H, Shaeri M. Encounter with violence against women in Islamic countries, Tehran, Iran. Hogogdan and Daneshnegar. 2006;15(2):9-123.

8. Ghazizadeh A. Domestic violence: a cross-sectional study in an Iranian city. East Mediterr Health J. 2005;11(5-6):880-7. [PubMed: 16761657]

9. Mikton C. Preventing intimate partner and sexual violence against women: taking action and generating evidence. Inj Prev. 2010;16(5):359-60. doi: 10.1136/ip.2010.029629. [PubMed: 20921563]

10. Lowe P, Humphreys C, Williams SJ. Night terrors: women's experiences of (not) sleeping where there is domestic violence. Violence Against Women. 2007;13(6):549-61. doi: 10.1177/1077801207301556. [PubMed: 17515405]

11. Josephs LL, Abel EM. Investigating the relationship between intimate partner violence and HIV risk-propensity in Black/Af-
rican-American women. J Family Violence. 2009;24(4):221-9. doi: 10.1007/s10896-009-9223-X.

12. Yoshihama M. Battered women's coping strategies and psychological distress: differences by immigration status. Am J Community Psychol. 2002;30(3):429-52. [PubMed:12054037]

13. Wu V, Huff $\mathrm{H}$, Bhandari M. Pattern of physical injury associated with intimate partner violence in women presenting to the emergency department: a systematic review and meta-analysis. Trauma Violence Abuse. 2010;11(2):71-82. doi:10.1177/1524838010367503. [PubMed: 20430799]

14. Salehi Fadardi J. A comparative study of anxiety, stress, and depression in physically abused and non-abused Iranian wives. Iranian J Psychiatry Behav Sci. 2009;3(2):15-24.

15. Roberts GL, Williams GM, Lawrence JM, Raphael B. How does domestic violence affect women's mental health? Women Health. 1998;28(1):117-29. [PubMed:10022060]

16. Houry D, Kemball R, Rhodes KV, Kaslow NJ. Intimate partner violence and mental health symptoms in African American female ED patients. Am J Emerg Med. 2006;24(4):444-50. doi: 10.1016/j. ajem.2005.12.026. [PubMed:16787803]

17. Weingourt R, Maruyama T, Sawada I, Yoshino J. Domestic violence and women's mental health in Japan. Int Nurs Rev. 2001;48(2):1028. [PubMed:11407455]

18. Avdibegovic E, Sinanovic O. Consequences of domestic violence on women's mental health in Bosnia and Herzegovina. Croat Med J. 2006;47(5):730-41. [PubMed:17042065]

19. Faramarzi M, Esmailzadeh S, Mosavi S. Prevalence and determinants of intimate partner violence in Babol City, Islamic Republic of Iran. East Mediterr Health J. 2005;11(5-6):870-9. [PubMed: 16761656]

20. Babu BV, Kar SK. Domestic violence against women in eastern India: a population-based study on prevalence and related issues. BMC Public Health. 2009;9:129. doi: 10.1186/1471-2458-9-129. [PubMed:19426515]

21. Rosen $\mathrm{KH}$, Bartle-Haring S, Stith SM. Using Bowen theory to enhance understanding of the intergenerational transmission of dating violence. J Family Issues. 2001;22(1):124-42. doi: 10.1177/019251301022001006.

22. Bonomi AE, Thompson RS, Anderson M, Reid RJ, Carrell D, Dimer JA, et al. Intimate partner violence and women's physical, mental, and social functioning. Am J Prev Med. 2006;30(6):458-66. doi:10.1016/j.amepre.2006.01.015. [PubMed: 16704938]

23. Salari Z, Nakhaee N. Identifying types of domestic violence and its associated risk factors in a pregnant population in Kerman hospitals, Iran Republic. Asia Pac J Public Health. 2008;20(1):4955. doi: 10.1177/1010539507308386. [PubMed: 19124298]

24. Babu BV, Kar SK. Domestic violence in Eastern India: factors associated with victimization and perpetration. Public Health. 2010;124(3):136-48. doi: 10.1016/j.puhe.2010.01.014. [PubMed: 20223489]

25. Jewkes R, Levin J, Penn-Kekana L. Risk factors for domestic violence: findings from a South African cross-sectional study. Soc Sci Med. 2002;55(9):1603-17. [PubMed:12297246]

26. Ghahhari S, Mazdarani S, Khalilian A, Zarghami M. Spouse abuse in Sari-Iran. Iran J Psychiatry Behav Sci . 2008;2(1):31-5.

27. Pournaghash-Tehrani S. Domestic violence in Iran: A literature review. Aggress Violent Beh. 2011;16(1):1-5. doi: 10.1016/j. avb.2010.12.001. 